Pacific Journal of Mathematics

EXTREME MARKOV OPERATORS AND THE ORBITS OF 


\section{EXTREME MARKOV OPERATORS AND THE ORBITS OF RYFF}

\section{RAY C. ShIFLETT}

Let $X$ be the unit interval with the Lebesgue structure and let $m$ be Lebesgue measure. A Markov operator with invariant measure $m$ is an operator $T$ on $L_{\infty}(X, m)$ such that $T 1=1$ and $\int T f d m=\int f d m$ for all $f$ in $L_{\infty}(X, m)$. If $\theta$ is a measure-preserving transformation on $X$, then $\hat{\theta} f=f \circ \theta$ defines a Markov operator. Each such $\hat{\theta}$ is an extreme point in the convex set of Markov operators.

Let $\Omega(f)$ be the set of all $g \in L_{1}(X, m)$ such that $T f=g$ for some Markov operator $T$. This convex set is called the orbit of $f$. The extreme points of $\Omega(f)$ are equimeasurable to $f$ and arise from Markov operators of the form $\hat{\theta} \hat{\sigma}^{*}$. This paper shows the connection between extreme points of the set of Markov operators and the extreme points of $\Omega(f)$. The set of Markov operators which carry $f$ to a given extreme point of $\Omega(f)$ is shown to contain an extreme Markov operator. The Markov operators of the from $\hat{\theta} \hat{\sigma}^{*}$ are shown to be extreme when $\theta$ is invertible. It is also shown that not all extreme operators factor into $\hat{\theta} \hat{\sigma}^{*}$ and that there are $\theta$ and $\sigma$ such that $\hat{\theta} \hat{\sigma}^{*}$ is not extreme.

This paper deals with the problem of extreme points in the convex set of Markov operators and how they relate to the extreme points of orbits of elements from $L_{1}$ as defined by J. V. Ryff. The author would like to express his gratitude to Professor J. V. Ryff for discussing this work with the author and to the referee for his helpful comments.

A Markov operator, with Lebesgue measure invariant, is an operator $T$ defined on $L_{\infty}(X, m)(X=[0,1]$ and $m$ is Lebesgue measure) which satisfies:

(1) $T$ is a positive operator

(2) $T 1=1$

(3) $\int_{x} f d m=\int_{x} T f d m$.

The norm of $T$, in the $L_{\infty}$ norm, is one. $T$ may be extended to $L_{1}$ such that $\|T\|_{1}=1$ and, using the Riesz convexity theorem, $T$ may be extended uniquely to $L_{p}$ as a contraction mapping for each $p, 1<p<\infty$. Therefore, $T$ is defined on the Hilbert space $L_{2}$ and the adjoint, $T^{*}$, is well defined.

If $\theta: X \rightarrow X$ is a measure-preserving transformation, that is, if $\theta$ is measurable and $m\left(\theta^{-1} A\right)=m(A)$ for every measurable $A$, then the operator defined by $\hat{\theta} f=f \circ \theta$ is an extreme point in the set of Markov 
operators. If $T$ is extreme, then $T^{*}$ is extreme. James R. Brown proved that the set of operators induced by invertible measure-preserving transformations was dense in the set of Markov operators in the weak operator topology on $L_{p}, 1<p<\infty$. He also proved that the set of Markov operators is the closed convex hull of the set of operators induced by invertible measure-preserving transformations in the strong operator topology [1].

There are examples of self-adjoint, extreme Markov operators which are not induced by a measure-preserving transformation. R. G. Douglas [3] and J. Lindenstrauss [5] gave, independently, the only known characterization of the extreme points of the set of Markov operators.

J. V. Ryff gave the following definition.

Definition. The orbit of $f \in L_{1}, \Omega(f)$, is the set of $g \in L_{1}$ where $g=T f$ for some Markov operator $T$.

Ryff's work, $[6,7,8]$, with these orbits suggests a possible connection between the extreme points of the set of Markov operators and the extreme points of $\Omega(f)$. The first theorem makes this connection explicit. Theorems 2,3 , and 4 give further clarification of this relationship. Theorems 5 and 6 show the limitation of this approach.

THEOREM 1. If $M_{f g}$ is the set of Markov operators which map $f$ to $g$ and if $g$ is an extreme point of $\Omega(f)$, then $M_{f g}$ contains an extreme point of the convex set of Markov operators.

Proof. Let $t T_{1}+(1-t) T_{2}$ be in $M_{f g}$ where $T_{1}$ and $T_{2}$ are Markov operators and $0<t<1$. Then $t T_{1} f+(1-t) T_{2} f=g$. Therefore, $T_{1} f=$ $T_{2} f=g$ since $g$ is given as extreme. Thus $M_{f g}$ is an extremal subset of the set of Markov operators.

The set of Markov operators is compact in the weak operator topology [1]. Now let $\left\langle T_{\alpha}\right\rangle$ be a net in $M_{f g}$ which converges to $T$ in the strong operator topogy; that is, $T_{\alpha} f$ converges to $T f$ for every $f$ in $L_{1}$. Thus $T f=g$ since $T_{\alpha} f=g$ for every $\alpha$. Therefore, $T \in M_{f g}$. This proves that $M_{f g}$ is closed in the strong operator topology. A convex set has the same closure in the weak operator topology as in the strong operator topology. Thus $M_{f g}$ is a closed, compact, convex, extremal subset of the set of Markov operators, which is a convex subset of a locally convex topological vector space. Thus $M_{f g}$ contains an extreme Markov operator, see page 67; [9].

Ryff characterized the extreme points of $\Omega(f)$ as those elements which are equimeasurable to $f$ [6]. These arise from Markov operators which may be written as $(\hat{\theta}) \circ(\hat{\sigma})^{*}$ where $\theta$ and $\sigma$ are measure-pre- 
serving transformations. Let $I_{A}$ bə the characteristic function of the set $A$.

THEOREM 2. If $\theta$ and $\sigma$ are measure-preserving transformations with $\theta$ invertible then $T=\hat{\theta} \hat{\sigma}^{*}$ is extreme.

Proof. $\theta$ is an invertible measure-preserving transformation if and only if $\hat{\theta}$ is unitary [1]. $\hat{\theta}$ is unitary if and only if $\hat{\theta}$ and $\hat{\theta}^{*}$ are isometries. Thus $\hat{\theta}^{-1}=\hat{\theta}^{*}$. Therefore, $\hat{\theta} \hat{\sigma}^{*}=\left(\hat{\sigma} \hat{\theta}^{-1}\right)^{*}$. Since $\hat{\sigma} \hat{\theta}^{-1} I_{B}=$ $I_{B}\left(\theta^{-1} \sigma\right)$ and $m\left(\left(\theta^{-1} \sigma\right)^{-1} B\right)=m(B)$, the operator $\hat{\theta} \hat{\sigma}^{*}$ is the adjoint of that operator induced by the measure-preserving transformation $\theta^{-1} \sigma$ and is therefore extreme.

THEOREM 3. If $\theta$ and $\sigma$ are measure-preserving transformations, then there is an $f \in L_{1}$ sush that $\hat{\theta} \hat{\sigma}^{*} f$ is extreme in $\Omega(f)$.

Proof. Let $A$ be given with $m(A)>0$ and lef $f$ be the characteristic function $\sigma^{-1} A$. Any $g$ which is extreme in $\Omega(f)$ must be equimeasurable to $f$ and is, therefore, essentially a characterictic function of a set $B$ with $m(B)=m(A)$. Let $C$ be a measurable set, then $\left(I_{C}, \hat{\sigma}^{*} f\right) \equiv$ $\left(\hat{\sigma} I_{C}, f\right)=\left(\hat{\sigma} I_{C}, \hat{\sigma} I_{A}\right)=\left(I_{C}, I_{A}\right)$. Thus $\hat{\sigma}^{*} f=I_{A}$. Therefore, $\hat{\theta} \hat{\sigma}^{*} f=$ $\hat{\theta} I_{A}=I_{A} \circ \theta$, which is the characteristic function of $\theta^{-1} A$ with $m\left(\theta^{-1} A\right)=$ $m(A)$.

It is well known that any Markov operator which carries characteristic functions to characteristic functions is extreme. The next theorem is a partial result to a conjecture suggested by the above fact and Theorem 3. The conjecture is that given an extreme Markov operator there is some measurable set $B$ such that $0<m(B)<1$ and such that $T I_{B}$ is extreme in $\Omega\left(I_{B}\right)$. This would say that $T I_{B}=I_{A}$ for some $A$ with $m(A)=m(B)$.

THeOREM 4. If $T$ is extreme then there is a set $B$ such that $0<m(B)<1$ and such that $T I_{B}=I_{A}+F$ where $m(A)>0$ and $F=0$ on $A$.

Proof. Suppose for every $B$, with $0<m(B)<1$, it is true that $0 \leqq T I_{B}<1[\mathrm{~m}]$-almost everywhere. Then $1 \geqq T I_{-B}>0[\mathrm{~m}]$-almost everywhere. But $-B$ is measurable with $0<m(-B)<1$ so that $0<T I_{-B}<1[\mathrm{~m}]$-almost everywhere. Thus, for every $B, 0<T I_{B}<1$ $[m]$-almost everywhere. This implies that $\left(I_{A}, T I_{B}\right)>0$ for every $A$ and $B$ with $m(A)>0$ and $m(B)>0$. By Theorem 2, [2], $T$ is not extreme.

After the discovery that the extreme points of $\Omega(f)$ were given 
by $\hat{\theta} \hat{\sigma}^{*} f$, the conjecture was made that the extreme points of the set of Markov operators are characterized by those operators which factor into $\hat{\theta} \hat{\sigma}^{*}$. The last two results of this paper answer this conjecture completely.

J. R. Brown proved that $\mu(A \times B)=\left(I_{A}, T I_{B}\right)$ gives a one to one correspondence between the set of doubly stochastic measures and Markov operators [1]. The notation $\mu_{0}$ will be used to denote the measure associated with $\hat{\theta}$. The measure for $\hat{\sigma}^{*}$ is denoted by $\mu_{\sigma}^{*}$ and for $\hat{\theta} \hat{\sigma}^{*}$ by $\mu$. The next technical result is needed for the last two theorems.

Proposition. $\mu_{\theta}(A \times B)=\mu\left(A \times \sigma^{-1} B\right)$ and $\mu_{\sigma}^{*}(A \times B)=\mu\left(\theta^{-1} A \times B\right)$.

Proof.

$$
\begin{aligned}
\mu_{0}(A \times B) & =\left(I_{A}, \hat{\theta} I_{B}\right)=\left(\hat{\sigma} \hat{\theta} * I_{A}, \hat{\sigma} I_{B}\right)=\left(\hat{\theta} * I_{A}, \hat{\sigma}^{*} I_{B} \circ \sigma\right) \\
& =\left(I_{A}, \hat{\theta} \hat{\sigma}^{*} I_{B} \circ \sigma\right)=\mu\left(A \times \sigma^{-1} B\right) .
\end{aligned}
$$

The other equality is established in a similar manner.

THEOREM 5. There are extreme Markov operators which are not of the form $\hat{\theta} \hat{\sigma}^{*}$.

Proof. Let $T$ be defined by

$$
\begin{aligned}
& T I_{B}=(1 / 2) I_{2 B+(1 / 3)} \text { if } B \subset[0,1 / 3] \text { and } \\
& T I_{B}=I_{(1 / 2)(B-(1 / 3))}+(1 / 2) I_{B} \text { if } B \subset[1 / 3,1] .
\end{aligned}
$$

Let $\mu$ be the associated doubly stochastic measure. It is easily seen that $1 / 3$ of the mass of $\mu$ is uniformly distributed over the sets

$$
\begin{aligned}
& \{(x, y): y=(1 / 2)(x-1 / 3)\} \cap([1 / 3,1] \times[0,1 / 3]), \\
& \{(x, y): y=x\} \cap([1 / 3,1] \times[1 / 3,1]) \text { and } \\
& \{(x, y): y=2 x+1 / 3\} \cap([0,1 / 3] \times[1 / 3,1]) .
\end{aligned}
$$

By Theorem 2, [2], $\mu$ is extreme. It can be shown that $T=T^{*}$ [4].

Suppose $T=\hat{\theta} \hat{\sigma}^{*}$. By the proposition, $\mu_{0}(B \times A)=\left(I_{B}, T I_{A} \circ \sigma\right)=$ $\left(T I_{B}, I_{A} \circ \sigma\right)=\left((1 / 2) I_{2 B+(1 / 3)}, I_{A} \circ \sigma\right)=(1 / 2) \mu_{\sigma}([2 B+(1 / 3)] \times A)$ for every $B \subset[0,1 / 3]$ and $A$ measurable. Also $\mu_{\theta}(B \times A)=m\left(B \cap \theta^{-1} A\right)$ and therefore,

$$
m\left(B \cap \theta^{-1} A\right)=(1 / 2) m\left([2 B+(1 / 3)] \cap\left(\sigma^{-1} A\right) .\right.
$$

By the proposition, $\mu_{\sigma}^{*}(A \times B)=\mu\left(\theta^{-1} A \times B\right)$. Similar manipulations as those yielding (1) will yield

$$
m\left(B \cap \sigma^{-1} A\right)=(1 / 2) m\left(\theta^{-1} A \cap[2 B+(1 / 3)]\right)
$$


for $B \subset[0,1 / 3]$ and $A$ measurable.

Let $C=2 B+(1 / 3) \subset[1 / 3,1]$. Then $\mu_{o}^{*}(A \times C)=\mu\left[\theta^{-1} A \times C\right]=$ $\left(I_{A} \circ \theta, T I_{C}\right)=\left(I_{A} \circ \theta, I_{(1 / 2)(C-(1 / 3))}\right)+\left(1 / 2\left(I_{A} \circ \theta, I_{C}\right)=m\left((1 / 2)[C-(1 / 3)] \cap \theta^{-1} A\right)+\right.$ $(1 / 2) m\left(C \cap^{-1} A\right)$. Therefore,

$$
\begin{aligned}
m\left([2 B+(1 / 3)] \cap \sigma^{-1} A\right)= & m\left(B \cap \theta^{-1} A\right)+(1 / 2) m([2 B \\
& \left.+(1 / 3)] \subset \theta^{-1} A\right) .
\end{aligned}
$$

Equations (1) and (3) yield

$$
m\left([2 B+(1 / 3)] \cap \sigma^{-1} A\right)=m\left([2 B+(1 / 3)] \cap \theta^{-1} A\right)
$$

for all measurable $B \subset[0,1 / 3]$ and all measurable $A$. Equations (2) and (4) yield

$$
(1 / 2) m\left([2 B+(1 / 3)] \cap \sigma^{-1} A\right)=m\left(B \cap \sigma^{-1} A\right)
$$

for $B \subset[0,1 / 3]$ and all $A$. Then (5) and (1) give

$$
m\left(B \cap \sigma^{-1} A\right)=m\left(B \cap \theta^{-1} A\right)
$$

for all measurable $B \subset[0,1 / 3]$ and all measurable $A$. Since every $C \subset[1 / 3,1]$ is the image of some $B \subset[0,1 / 3]$ under $2 B+(1 / 3)$, for any measurable $A$ and $C, \mu_{\theta}(A \times C)=\mu_{\sigma}(A \times C)$. Thus $\mu_{\theta}=\mu_{\sigma}$ and $\theta=\sigma$.

Thus, if $T=\hat{\theta} \hat{\sigma}^{*}$, it must be that $T=\hat{\theta} \hat{\theta}^{*}$. However,

$$
\left(I_{[0,1 / 3]}, T I_{[0,1 / 3]}\right)=(1 / 2)\left(I_{[0,1 / 3]}, I_{[1 / 3,1]}\right)=0 \text {. }
$$

Then $\left(I_{[0,1 / 3]}, \hat{\theta} \hat{\theta}^{*} I_{[0,1 / 3]}\right)=\left(\hat{\theta}^{*} I_{[0,1 / 3]}, \hat{\theta}^{*} I_{[0,1 / 3]}\right)=\int_{X}\left(\hat{\theta}^{*} I_{[0,1 / 3]}\right)^{2} d m=0 . \quad$ Then $\hat{\theta}^{*} I_{[0,1 / 3]}=0[m]$-almost everywhere. This says that $\left(1, \hat{\theta}^{*} I_{[0,1 / 3]}\right)=0$, which is a contradiction. Thus $T$ is not of the form $\hat{\theta} \hat{\sigma}^{*}$.

THEOREM 6. There are operators $T=\hat{\theta} \hat{\sigma}^{*}$ which are not extreme.

Proof. Let $\sigma(x)=2 x(\bmod 1)$ and $\theta(x)=3 x$ if $x \in[0,1 / 3]$ and $\theta(x)=$ $(1 / 2)(3 x-1)$ if $x \in[1 / 3,1]$. For any

$$
f \in L_{2},\left(\hat{\sigma}^{*} f\right)(x)=(1 / 2) f(x / 2)+(1 / 2) f((x+1) / 2) \text {. }
$$

Thus

$$
\begin{aligned}
\mu(A \times B)=\left(I_{A}, T I_{B}\right)= & \left(I_{A}, \hat{\theta}\left[(1 / 2) I_{2 B}+(1 / 2) I_{2 B-1}\right]\right) \\
= & (1 / 2) \int_{[0,1 / 3]} I_{A}(x) I_{2 B}(3 x) m(d x) \\
& +(1 / 2) \int_{[1 / 31]} I_{A}(x) I_{2 B}((3 x-1) / 2) m(d x) \\
& +(1 / 2) \int_{[0,1 / 3]} I_{A}(x) I_{2 B-1}(3 x) m(d x) \\
& +(1 / 2) \int_{[1 / 3,1]} I_{A}(x) I_{2 B-1}((3 x-1) / 2) m(d x) .
\end{aligned}
$$


If $B \subset[0,1 / 2]$, this equality will yield

$$
\mu(A \times B)=(1 / 2) m(A \cap(1 / 3)[2 B \cup(4 B+1)]) .
$$

If $B \subset[1 / 2,1], \mu(A \times B)=(1 / 2) m(A \cap(1 / 3)[(2 B-1) \cup(4 B-1)])$. So, for $A \subset[0,1 / 3]$ and $B \subset[0,1 / 2], \mu(A \times B)=(1 / 2) m[A \cap(2 B) / 3]$. Then $1 / 6$ of the mass of $\mu$ is distributed uniformly on $y=(3 / 2) x$ in $[0,1 / 3] \times$ $[0,1 / 2]$.

Similar manipulations show that the $1 / 3$ of the mass is on $y=$ $(3 / 4)(x-(1 / 4))$ in $[1 / 3,1] \times[0,1 / 2]$ and $1 / 6$ on $y=(3 / 2) x+(1 / 2)$ in $[0,1 / 3] \times[1 / 2,1]$ and $1 / 3$ on $y=(3 / 4) x+(1 / 4)$ in $[1 / 3,1] \times[1 / 2,1]$. By Theorem 1, [2], this $T$ is not extreme.

Theorem 5 does not answer the more general conjecture, which the author made, that every extreme Markov operator factors into a product of operators induced by measure preserving transformations and the adjoints of such operators. The author has not been able to answer this question. It is easy to show that this conjecture could be stated as every extreme Markov operator $T$ may be written as $T=T_{1} T_{2} \cdots T_{n}$ where $T_{i}=\hat{\theta}_{i} \hat{\sigma}_{i *}\left(\theta_{1}\right.$ or $\sigma_{n}$ may be the identity transformation). Theorem 6 shows this property can not characterize the extreme points.

\section{REFERENCES}

1. J. R. Brown, Approximation theorems for Markov operators, Pacific J. Math., 16 (1966), 13-23.

2. J. R. Brown and R. C. Shiflett, On extreme doubly stochastic measures, Michigan Math. J., 17 (1970), 249-254.

3. R. G. Douglas, On extremal measures and subspace density, Michigan Math. J., 11 (1964), 243-246.

4. R. E. Jaffa, Extremal Doubly Stochastic Operators and Related Matrices, Ph. D. thesis. Berkeley, University of California, 1964. 53p.

5. J. Lindenstrauss, A remark on extreme doubly stochastic measures, Amer. Math. Monthly, 72 (1965), 379-382.

6. J. V. Ryff, Extreme points of some convex subsets of $L^{1}(0,1)$, Proc. Amer. Math. Soc., 18 (1967), 1026-1034.

7. - On the representation of doubly stochastic operators, Pacific J. Math., 13 (1963), 1379-1386.

8. - Orbits of $L^{1}$-functions under doubly stochastic transformations, Trans. Amer. Math. Soc., 117 (1965), 92-100.

9. H. H. Schaefer, Topological Vector Spaces, New York, Macmillan, 1966.

10. R. C. Shiflett, On extreme doubly stochastic measures and Feldman's conjecture, Tech. Report 39, Oregon State University Press, 1968.

Received April 30, 1970. This paper contains a portion of the author's dissertation presented for the degree of Doctor of Philosophy at Oregon State University and was supported by U. S. National Science Foundation Grants in Applied Analysis, GP-6144 and GP-7474. 


\section{PACIFIC JOURNAL OF MATHEMATICS}

\section{EDITORS}

H. SAMELSON

Stanford University

Stanford, California 94305

C. R. HOBBY

University of Washington

Seattle, Washington 98105
J. DugundJI

Department of Mathematics

University of Southern California

Los Angeles, California 90007

RICHARD ARENS

University of California

Los Angeles, California 90024

\section{ASSOCIATE EDITORS}

E. F. BeCKENBACH

B. H. NeumanN

F. WOLF

K. YosHIDA

\section{SUPPORTING INSTITUTIONS}

UNIVERSITY OF BRITISH COLUMBIA

CALIFORNIA INSTITUTE OF TECHNOLOGY

UNIVERSITY OF CALIFORNIA

MONTANA STATE UNIVERSITY

UNIVERSITY OF NEVADA

NEW MEXICO STATE UNIVERSITY

OREGON STATE UNIVERSITY

UNIVERSITY OF OREGON

OSARA UNIVERSITY
UNIVERSITY OF SOUTHERN CALIFORNIA STANFORD UNIVERSITY

UNIVERSITY OF TOKYO

UNIVERSITY OF UTAH

WASHINGTON STATE UNIVERSITY

UNIVERSITY OF WASHINGTON

AMERICAN MATHEMATICAL SOCIETY

NAVAL WEAPONS CENTER

Printed in Japan by International Academic Printing Co., Ltd., Tokyo, Japan 


\section{Pacific Journal of Mathematics}

\section{Vol. 40, No. $1 \quad$ September, 1972}

Alex Bacopoulos and Athanassios G. Kartsatos, On polynomials

approximating the solutions of nonlinear differential equations........

Monte Boisen and Max Dean Larsen, Prüfer and valuation rings with zero

divisors ..........................................

James J. Bowe, Neat homomorphisms

David W. Boyd and Hershy Kisilevsky, The Diophantine equation

$$
u(u+1)(u+2)(u+3)=v(v+1)(v+2) \ldots \ldots \ldots \ldots \ldots \ldots \ldots
$$

George Ulrich Brauer, Summability and Fourier analysis ...............

Robin B. S. Brooks, On removing coincidences of two maps when only one,

rather than both, of them may be deformed by a homotopy ............

Frank Castagna and Geert Caleb Ernst Prins, Every generalized Petersen

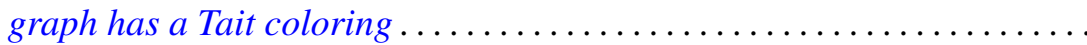

Micheal Neal Dyer, Rational homology and Whitehead products ..........

John Fuelberth and Mark Lawrence Teply, The singular submodule of a

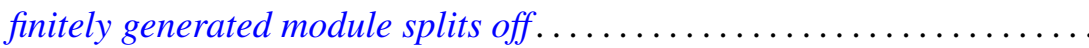

Robert Gold, $\Gamma$-extensions of imaginary quadratic fields ............ 83

Myron Goldberg and John W. Moon, Cycles in k-strong tournaments.......

Darald Joe Hartfiel and J. W. Spellmann, Diagonal similarity of irreducible

matrices to row stochastic matrices...............

Wayland M. Hubbart, Some results on blocks over local fields ..

Alan Loeb Kostinsky, Projective lattices and bounded homomorphisms....

Kenneth O. Leland, Maximum modulus theorems for algebras of operator

valued functions ...

Jerome Irving Malitz and William Nelson Reinhardt, Maximal models in the

language with quantifier "there exist uncountably many" ..

John Douglas Moore, Isometric immersions of space forms in space

forms.

Ronald C. Mullin and Ralph Gordon Stanton, A map-theoretic approach to

Davenport-Schinzel sequences ....................

Chull Park, On Fredholm transformations in Yeh-Wiener space. .

Stanley Poreda, Complex Chebyshev alterations ..............

Ray C. Shiflett, Extreme Markov operators and the orbits of Ryff. ...

Robert L. Snider, Lattices of radicals .....................

Ralph Richard Summerhill, Unknotting cones in the topological

category ................................

Charles Irvin Vinsonhaler, A note on two generalizations of $\mathrm{QF}-3 \ldots \ldots 229$

William Patterson Wardlaw, Defining relations for certain integrally

parameterized Chevalley groups...................

William Jennings Wickless, Abelian groups which admit only nilpotent

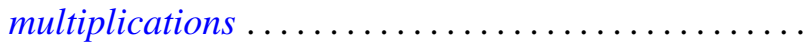

